This item was submitted to Loughborough's Research Repository by the author.

Items in Figshare are protected by copyright, with all rights reserved, unless otherwise indicated.

\title{
Determining spectral response of a photovoltaic device using polychromatic
}

\section{filters}

PLEASE CITE THE PUBLISHED VERSION

http://dx.doi.org/10.1049/iet-rpg.2013.0248

\section{PUBLISHER}

(C) The Institution of Engineering and Technology (IET)

\section{VERSION}

VoR (Version of Record)

\section{PUBLISHER STATEMENT}

This work is made available according to the conditions of the Creative Commons Attribution 3.0 Unported (CC BY 3.0) licence. Full details of this licence are available at: http://creativecommons.org/licenses/by/3.0/

\section{LICENCE}

CC BY 3.0

\section{REPOSITORY RECORD}

Sara, Ira D., Thomas R. Betts, and Ralph Gottschalg. 2019. "Determining Spectral Response of a Photovoltaic Device Using Polychromatic Filters". figshare. https://hdl.handle.net/2134/16266. 


\section{Open access notice for the paper 'Determining Spectral Response of a Photovoltaic Device using Polychromatic Filters'}

The paper Sara, I.D., Betts, T.R., Gottschalg, R.: 'Determining spectral response of a photovoltaic device using polychromatic filters'. IET Renewable Power Generation, 2014, 8, (5), pp. 467-473 is now available as open access and free to view at http://digital-library.theiet. org/content/journals/10.1049/iet-rpg.2013.0248. Its previous copyright status has been superseded by the Creative Commons Attribution Licence (http://creativecommons.org/ licenses/by/3.0/).

doi: 10.1049/iet-rpg.2014.0323 


\title{
Determining spectral response of a photovoltaic device using polychromatic filters
}

\author{
Ira Devi Sara1,2, Thomas R. Betts ${ }^{1}$, Ralph Gottschalg ${ }^{1}$ \\ ${ }^{1}$ Centre for Renewable Energy Systems Technology (CREST), School of Electronic, Electrical and Systems Engineering, \\ Loughborough University, LE11 3TU, UK \\ ${ }^{2}$ Department of Electrical Engineering, Syiah Kuala University, Darussalam, Banda Aceh, 23111, Indonesia \\ E-mail: I.D.Sara@Iboro.ac.uk
}

\begin{abstract}
This study introduces a new method for determining the spectral response of a photovoltaic (PV) device. Instead of illuminating the PV device under test with monochromatic beams at different wavelengths, the device is irradiated with different broadband spectra. Variations in the spectra are made by employing different types of polychromatic filters. The advantage of using these types of filters is the increase in the light intensity incident on the measurement plane, compared with narrowband filtered light. As a consequence, the spectral response measurement setup becomes very simple, comprising only a light source, a set of polychromatic filters, spectroradiometer and source-meter unit. The spectral response is determined by fitting the short-circuit currents measured under different spectra to a chosen spectral response model. The proposed method is verified by comparing the results to spectral response curves measured with a traditional monochromatic method, which show excellent agreement. From this analysis, it can be concluded that the proposed method is feasible as a new technique for determining the spectral response of a PV device.
\end{abstract}

\section{Introduction}

The spectral response (SR) of a photovoltaic (PV) device is one of the key characteristics used to determine device material and junction quality during cell analysis [1]. Further applications are in performance characterisation, such as in the process of choosing an appropriate reference PV device, tuning solar simulator light intensity and correcting the measured current and voltage $(I-V)$ curve to be equivalent to Standard Test Conditions (STC) and accommodate for any residual spectral mismatch [2-4]. The spectral response of a PV device is determined traditionally by illuminating the device with a series of (quasi-) monochromatic beams at different wavelengths and measuring the short-circuit current generated under each wavelength [5]. This technique for spectral response determination is known as the monochromatic filter method and the procedure of how to perform measurements on a single-junction PV device is described in the international standard IEC 60904-8: measurement of the spectral response of a PV device [6]. As stated in the IEC standard, the monochromatic beams radiating onto a PV device under test (DUT) should have a narrow transmission bandwidth and cover the entire range of spectral interest of the DUT with wavelength steps of less than $50 \mathrm{~nm}$. To obtain accurate spectral response determinations, the intensity of monochromatic beams must be sufficient to generate measurable response signals from the DUT, be spatially uniform and temporally stable during the measurement. A set of interference filters with narrow bandwidths is generally preferable to a grating monochromator for measuring larger devices, because of the larger irradiances and hence currents [7]. Further important conditions need to be met during the spectral response measurement to ensure consistently accurate results: Device temperature must be kept stable (ideally at $25^{\circ} \mathrm{C}$ ) as the spectral response of certain types of device changes with temperature, and the total incident irradiance must be set so as to operate the device in a region of linear current response [8, 9]. Changes in these conditions can lead to systematic errors in the spectral response determinations [10]. To overcome the non-linearity problem and to eliminate errors in the spectral response measurement because of trap state recombination within a PV device at low irradiance levels, bias lighting is generally applied for measuring spectral response, even of single-junction PV devices. The bias lighting functions to saturate the defect states of the PV device under test to produce high minority carrier densities [11, 12]. In the majority of installations, a lock-in technique is used to detect the monochromatic signals from other signals at a reference frequency that is provided by a chopper or an oscillator.

There are some challenges associated with the application of narrow bandwidth monochromatic filters for spectral response measurement, predominantly stray light effects in the measurement system, shape and width errors in the transmission pass band [13] and lack of flexibility in choosing a precise wavelength of interest [14]. The width errors are produced when narrow bandpass filters with asymmetric transmittance around their centre wavelength 


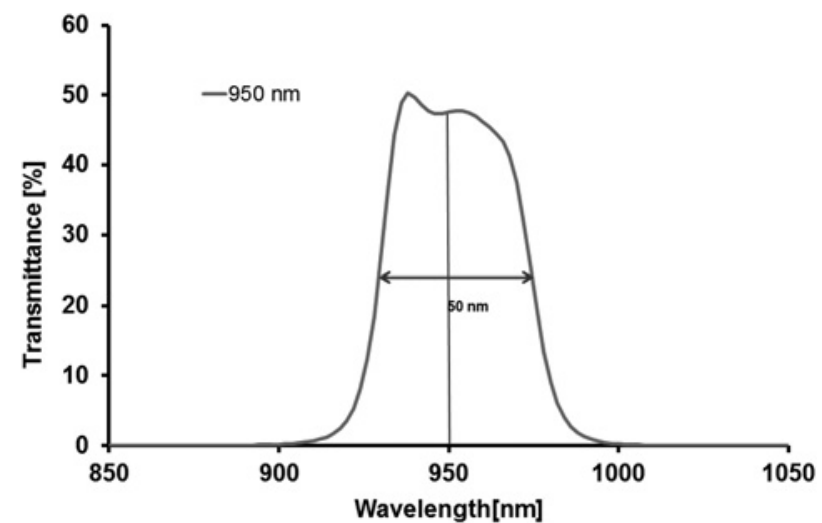

Fig. 1 Spectral transmittance of a narrow bandpass filter with a bandwidth of $50 \mathrm{~nm}$ full-width at half-maximum, measured at CREST

are used to filter the spectrum of a light source as shown in Fig. 1.

As shown in Fig. 1, the area under the filter transmittance curve is asymmetric and the quasi-monochromatic beam transmitted through this filter produces an unequal response current signal around the centre wavelength. As a result, the response signal is not mapped clearly to a given wavelength. These errors become prominent if the bandwidth of the asymmetric filter is greater than $10 \mathrm{~nm}$ [13]. Reducing the bandwidth of the filters reduces these errors, but also leads to a lower level of irradiance and limits the illumination area [15]. If a filter with a narrow bandwidth is used to filter the source light spectrum in the ultraviolet (UV) or near infrared ranges, where the response signal generated by the DUT is very weak, the response signal becomes prone to uncertainty because of reduced signal-to-noise. Therefore a phase lock-in technique is required to extract the monochromatic light response signal from background noise. Yet a powerful light source is still required to produce reasonable irradiance levels on a large area when filtered to narrow spectral bands. This makes monochromatic methods more suitable for measuring small area PV devices only, as the signals from large area devices are too weak to detect within acceptable uncertainty.

This paper introduces a new method for determining the spectral response of a PV device, a polychromatic filter method. The advantage of this method is that the beam intensity illuminating the DUT becomes higher than when using monochromatic filters. As a consequence, lock-in techniques and bias lighting are not required. This novel approach to obtain the spectral response is discussed in more detail in the following section.

\section{Spectral response measurement using polychromatic filters}

To obtain high accuracy in the spectral response measurement using monochromatic filters, a large number of narrow bandpass filters are required in order to obtain a high-resolution spectral response curve $[16,17]$. Employing a large number of specialised filters increases the cost of a spectral response measurement system. This build cost can be reduced by limiting the number of filters used and selecting cheaper filter materials. The polychromatic filters selected here meet these requirements as well as improving the accuracy in the spectral response measurement by providing higher light intensity on the test area by utilising filters with broad spectral bandwidth and employing only a few measurement instruments in its measurement system, which will be shown in the following section. As a result, the measurement errors that propagate through each instrument to the spectral response determination can be reduced. In addition, the response signal generated by a tested PV device at a given filter is not measured at a particular wavelength spectrum. This can eliminate the shape and width errors as encountered in the monochromatic filter method. A small number of filters with different spectral transmittance that is utilised in the measurement system and their cheap material costs have a positive impact on lowering the capital costs for building the spectral response measurement system. A set of five-identical sheets of the selected polychromatic filters, which are made from the polymeric material with a dimension of $50 \mathrm{~cm}$ by $60 \mathrm{~cm}$ only, costs at $£ 10.50$. This is almost 18 times cheaper than the cost of one glass optical filter with diameter of $25 \mathrm{~mm}$. In addition, by eliminating the use of the lock-in amplifier, the chopper and the bias lighting system in the spectral response measurement system, the building cost of this polychromatic filter method for the spectral response determination becomes much cheaper than that of the monochromatic filter method.

The polychromatic filter method contains a model of the spectral response at its core. The parameters of the model are determined through a fitting technique, based on an input dataset comprising the measured (or known) absolute spectral irradiance of several broadband spectra and the corresponding measured photocurrent generated by the DUT. Here, the spectra are generated from the light output of a stable xenon lamp, filtered sequentially by different polychromatic filters with broad transmittance bandwidths as shown in Fig. 2.

The goodness-of-fit used in the fitting technique is based on comparison of the measured currents with that derived from the measured spectra and spectral response model via the photocurrent formula

$$
I_{\mathrm{ph}}=A \cdot \int_{\lambda_{1}}^{\lambda_{2}} \operatorname{SR}(\lambda) \cdot E(\lambda) \mathrm{d} \lambda
$$

where $I_{\mathrm{ph}}$ is the photocurrent in amperes, SR is the spectral response of a tested $\mathrm{PV}$ device in $\mathrm{AW}^{-1}, E$ is the spectrum of the light source in $\mathrm{Wm}^{-2} \mathrm{~nm}^{-1}, \lambda_{1}$ and $\lambda_{2}$ are the extents

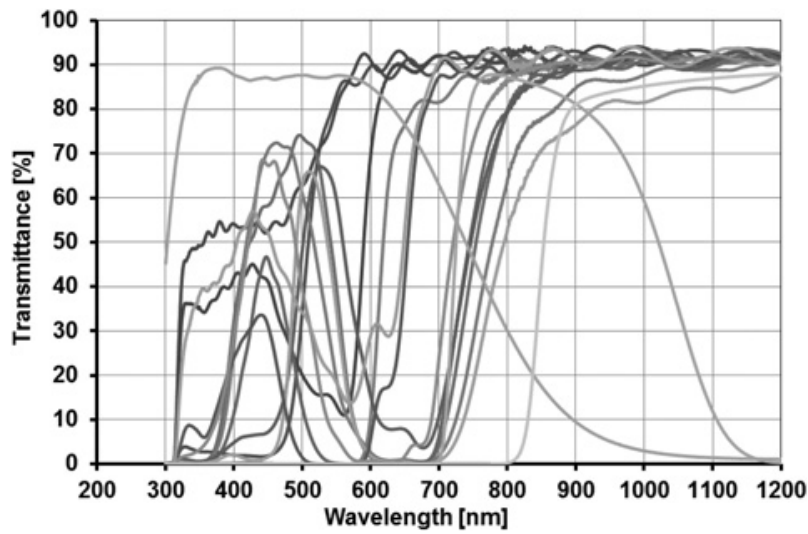

Fig. 2 Spectral transmittances of selected polychromatic filters for spectral response measurement 
of the wavelength range of PV device spectral response and $A$ is the area of the test device in $\mathrm{m}^{2}$.

The only unknown in the right-hand side of (1) is the spectral response of the DUT. To determine the unknown spectral response, a model for it must be assumed. In principle, any parameterised model could be used. In this work, a purely empirical model based on a fifth-order Gaussian function has been chosen for its flexibility to represent the spectral response of different PV devices tested, while requiring no prior knowledge of the device physics or structural detail [18]. The underlying equation is given in the following

$$
\mathrm{SR}(\lambda)=\sum_{i=1}^{5} a_{i} \exp \left[-\left(\frac{\lambda-b_{i}}{c_{i}}\right)^{2}\right]
$$

where $a_{i}, b_{i}$ and $c_{i}$ are the coefficients of the Gaussian model to be fitted.

The spectral response of a PV device is represented as a continuous curve, based on the above model. The fitting algorithm is developed to work iteratively to tune the coefficients to minimise the deviation of photocurrents measured under different spectral conditions to the photocurrent calculated from the formulae in (1) and (2). The final output of this fitting approach is a set of the appropriate fitted coefficients. By inputting these fitted values to the chosen spectral response model, the spectral response of the DUT is obtained. The entire fitting process is shown in Fig. 3.

As shown in Fig. 3, the fitting algorithm requires four input components:
1. A set of measured photocurrents.

2. A set of spectra corresponding to the measured photocurrents.

3. A suitable spectral response model and

4. Initial coefficient values for the chosen spectral response model.

\section{Experimental description}

The components used in the proposed spectral response method consist of a $100 \mathrm{~W}$ xenon arc lamp, a set of polychromatic filters to vary the spectra irradiating the tested PV device, a shutter to open and block the light source for accurate current measurement, a spectroradiometer to measure spectra and a source-meter unit to keep the tested PV device operating at its shortcircuit point and to measure the photocurrent generated.

The filters in the measurement system are a combination of two types: a small number of specialist glass filters and then the majority are lighting gel filters which are made from a thin polymeric material. The filters are shown in Fig. 4 in their mounting, which replaces the monochromatic filter block in CREST's QE measurement system. The mounting block includes many additional filters not used in the final configuration of the system for testing purposes. This final combination provides the desired variations across different wavelengths as shown in Fig. 2. The gel filters are stock equipment, usually used to provide colour lighting and correction in theatre and photography applications. Since the filters are exposed directly to the light source, a heat resistance test was performed to examine the deformation point of the lighting gel filters. The result showed that the

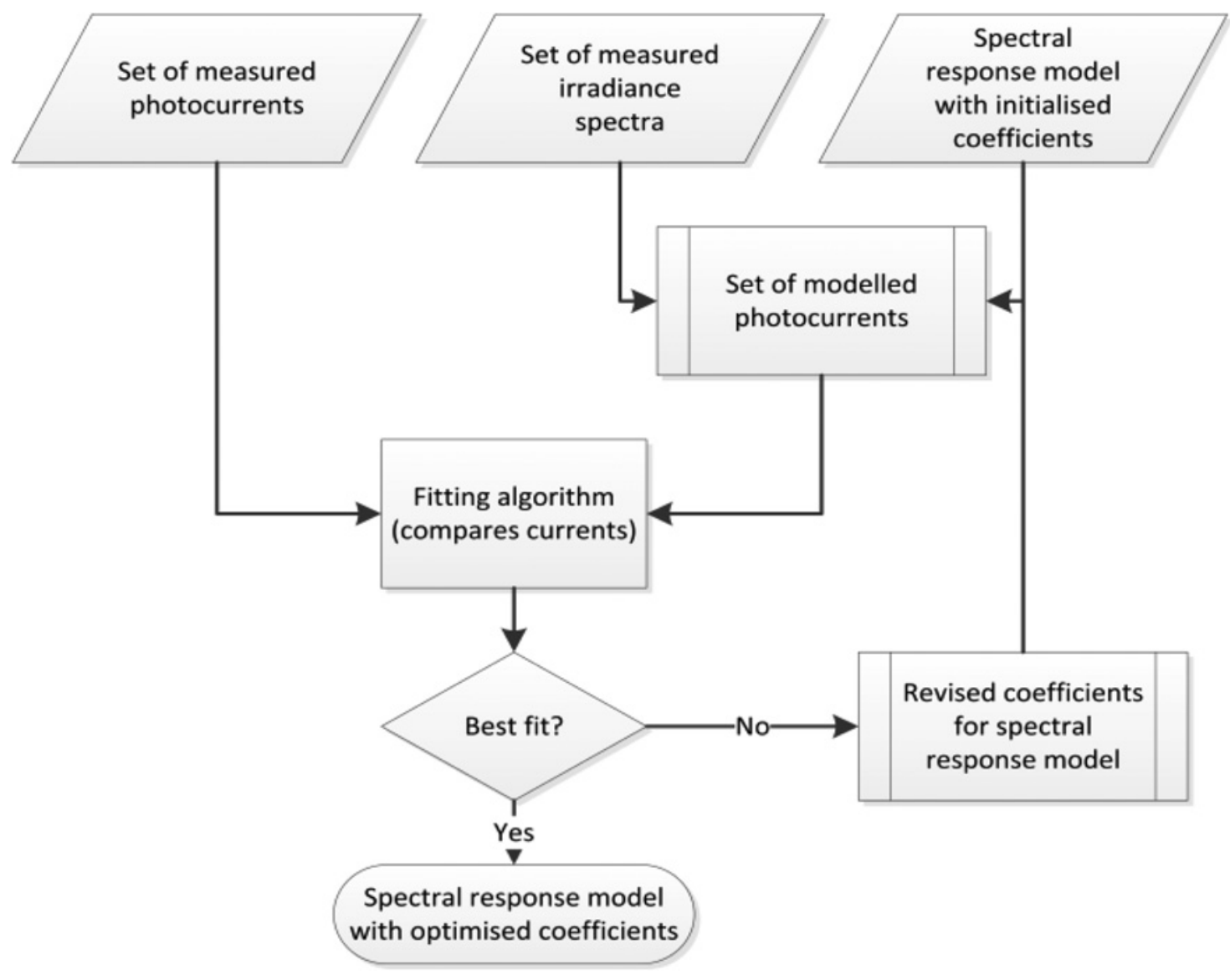

Fig. 3 Overview of polychromatic filter method to determine spectral response 


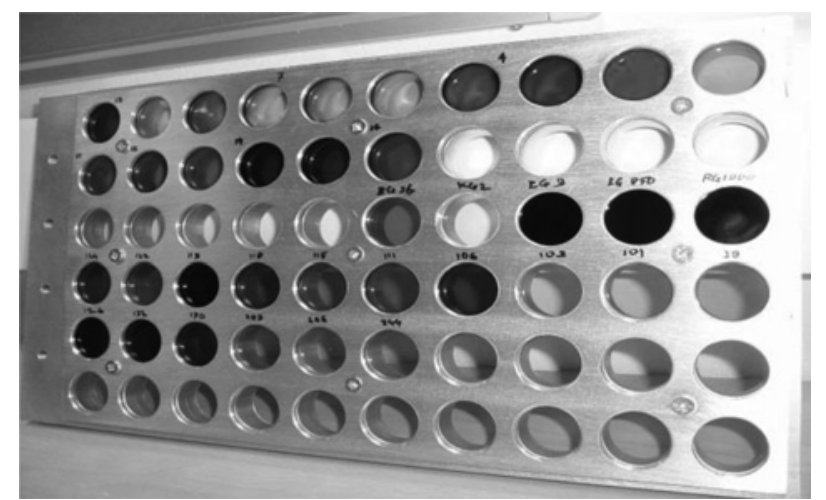

Fig. 4 Types of polychromatic filters used in the proposed spectral response method

filters can withstand temperatures up to $200^{\circ} \mathrm{C}$ for at least $2 \mathrm{~h}$ and indicates they are suitable to be employed in the spectral response measurement system.

The arrangement of the instruments used in the proposed spectral response method can be seen in Fig. 5.

The method was tested theoretically by carrying out simulations and selecting an appropriate sub-set of filters. In a second step, the model is validated practically by measuring three different PV devices, one mono-crystalline silicon (mono-c-Si), one poly-crystalline (poly-c-Si) and one amorphous silicon (a-Si). Prior to using the new spectral response method, the baseline spectral response curves of the selected PV devices were measured using the same equipment in the conventional monochromatic mode which has been validated against measurements taken in other laboratories by means of round robin intercomparisons. The measurement system employs a set of 44 narrow bandpass filters, a reference photodiode to measure the irradiance level falling onto the tested PV device and lock-in amplifiers to detect the signals generated by the tested PV device and the reference diode [19]. The mono-c-Si device has had its spectral response measured at an accredited PV calibration laboratory as an external validation. The spectral response determinations using the monochromatic filter method are shown in Fig. 6.

Having the measured spectral response curve, the next step is to measure the PV devices' photocurrents under various spectral conditions that are provided by the polychromatic filters. The photocurrent is measured at zero voltage bias [20]. The setup of this measurement is shown in Fig. 5. The measurement produces a set of photocurrents and their

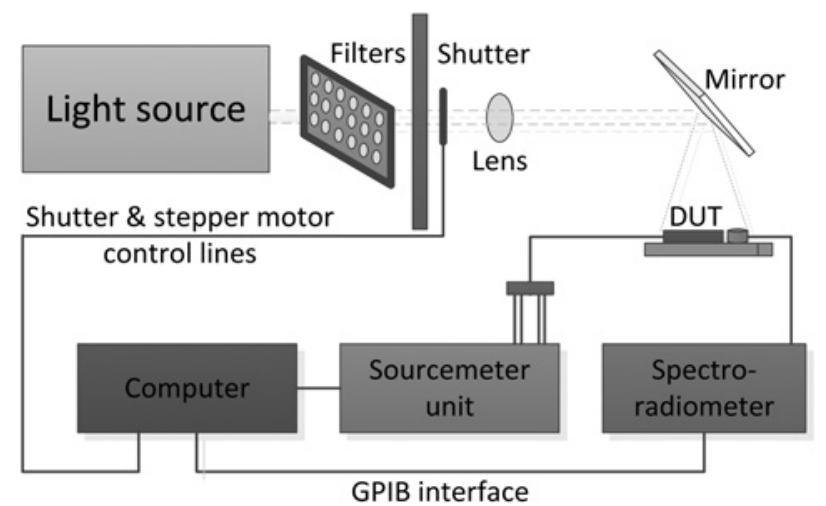

Fig. 5 Experimental setup of a new proposed spectral response method

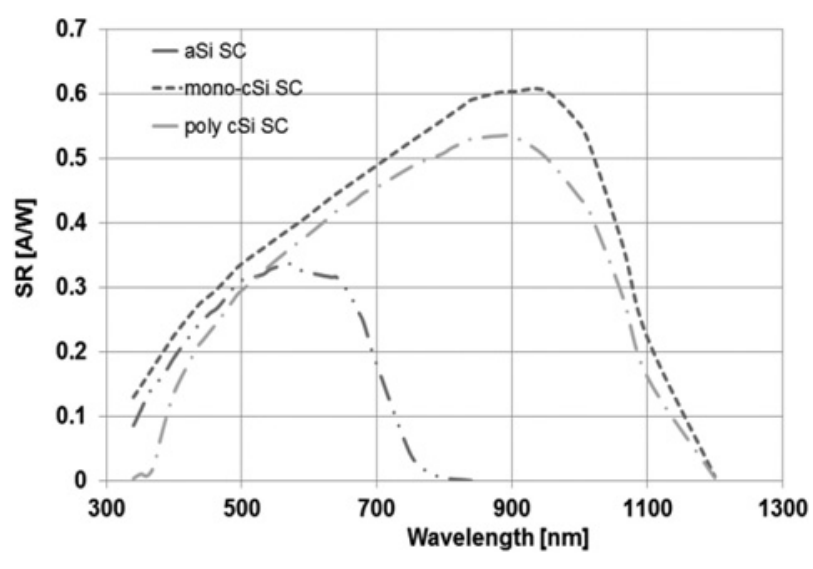

Fig. 6 Spectral response of selected PV devices measured using the conventional monochromatic filter method

corresponding spectra. These two sets of results are inputted to the fitting algorithm and by comparing the short-circuit currents obtained from the measurement and the photocurrent formula, the fitting algorithm returns the appropriate coefficients for the chosen spectral response model. Lastly these fitted coefficients are substituted into the spectral response model to obtain the spectral response curve of the DUT.

\section{Validation results}

The spectral response of each device obtained using the proposed polychromatic filter method is shown in the following figures, in direct comparison to the results from the standard spectral response measurement method (the monochromatic filter method).

There is only very slight difference between the spectral response curves of the mono-crystalline silicon device measured at CREST and at the calibration laboratory. This confirms the monochromatic spectral response measurements at CREST are credible. As can be seen in Fig. 7, the spectral response curve obtained using the polychromatic filter method is a very good qualitative match to those from the monochromatic filter method. The quantitative agreement by wavelength between the two measurement methods can be seen in Fig. 8. This figure shows that deviations in the spectral response measurement points across the given wavelengths are minimal, overall

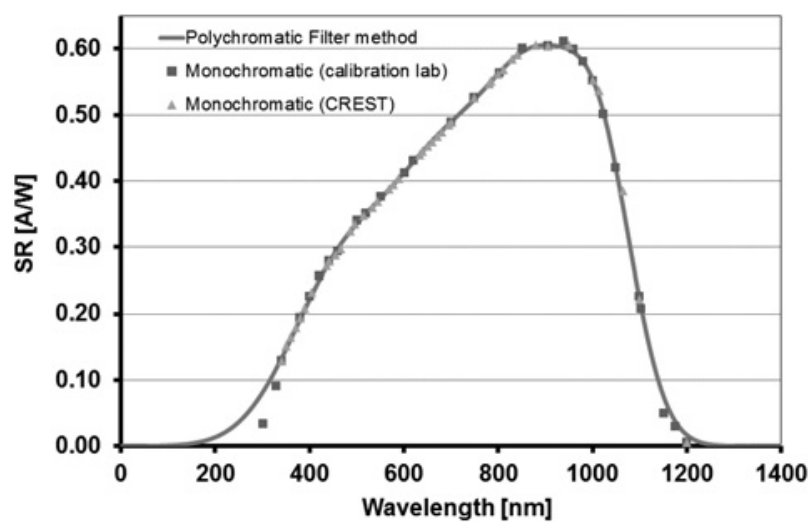

Fig. 7 Spectral response of a mono-c-Si device using different methods: the monochromatic filter method and the proposed polychromatic filter method 


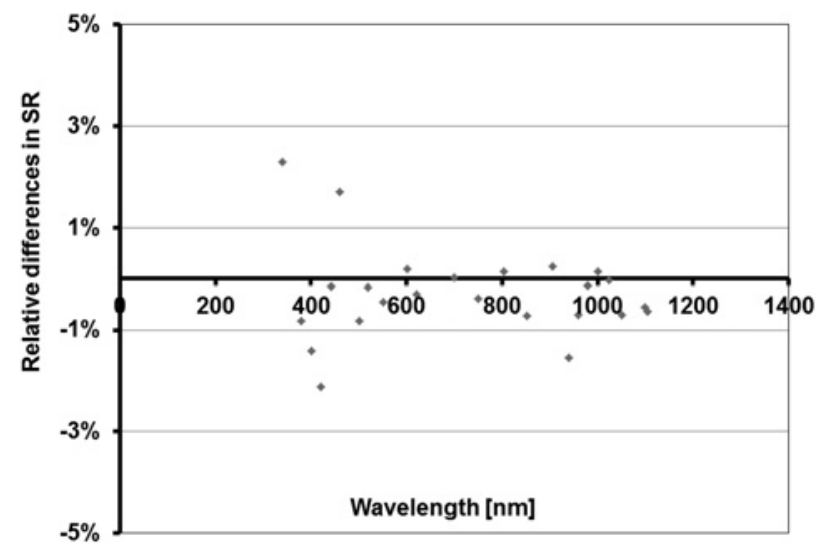

Fig. 8 Relative difference in spectral response of a mono-c-Si device using the polychromatic filter method, compared to the monochromatic measurement

within $\pm 2 \%$ which is slightly lower than the uncertainty in current measurement alone. Good agreement is also found for the poly-c-Si device as shown in Figs. 9 and 10 with relative differences within $\pm 5 \%$ for significant wavelengths (the UV and infrared regions). Higher deviations in these measurement points are caused by low levels of signal-to-noise ratio, yet contributions from these areas make little contribution to the global photocurrent.

The feasibility of the proposed method for determining the spectral response of an amorphous silicon device can be seen

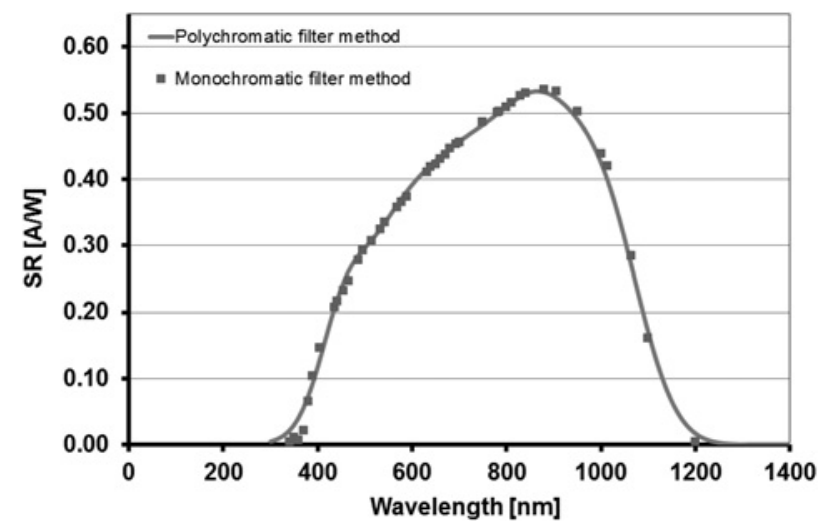

Fig. 9 Spectral response of a poly-c-Si device using two different methods: the polychromatic and monochromatic filter methods

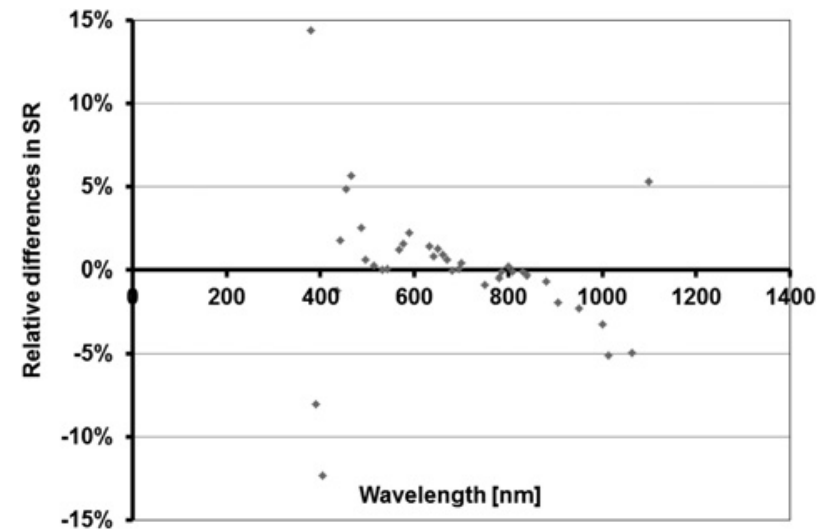

Fig. 10 Relative difference in spectral response of a poly-c-Si device, compared to the monochromatic measurement

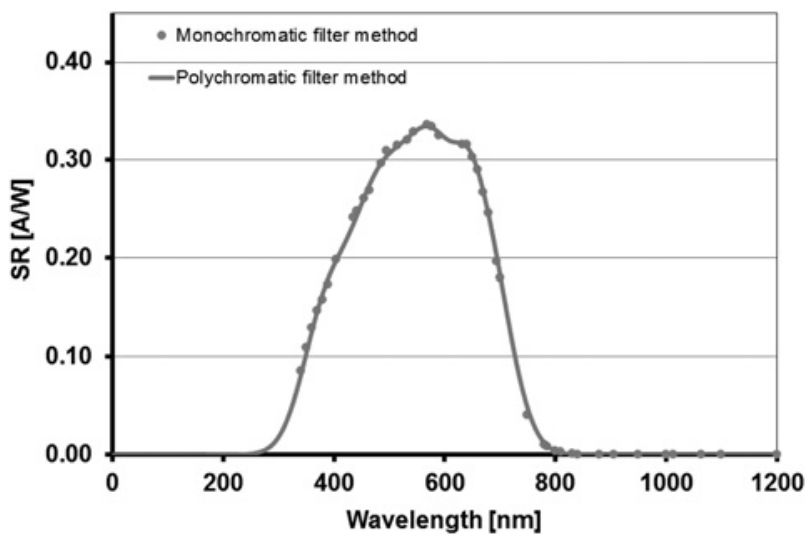

Fig. 11 Spectral response of an a-Si device using two different spectral response measurement methods: the polychromatic and monochromatic filter methods

in Fig. 11. Again, the spectral response curve obtained using the polychromatic filter method has an excellent match to that of using the monochromatic filter method with deviations across the given wavelengths of $\pm 2 \%$ as shown in Fig. 12 . It should be noted that all features, including the rather localised peak in the SR, are represented very well by the polychromatic measurements.

As the proposed spectral response determination method is empirical and works on fitting, its accuracy depends considerably on the accuracy of the measured short-circuit currents and irradiance spectra as well as the choice of the initial coefficient values of the spectral response model. The effects of accuracy of those input parameters on the spectral response determination can be seen from a sensitivity analysis using a Monte Carlo approach. In this technique, each input parameter is randomly varied within certain uncertainty levels, that is, $1,5,10$ and $20 \%$. The random numbers of the input parameters are drawn for 10000 cases from Gaussian probability distribution functions. For the measured spectra, the random numbers are generated as a function of wavelength at defined uncertainty levels. The random numbers for the initial values are made by varying a set of initial values that have been used in the fitting algorithm to produce a well-matched spectral response curve. When one input is varied, the others are kept constant at their optimised values. By doing this, the

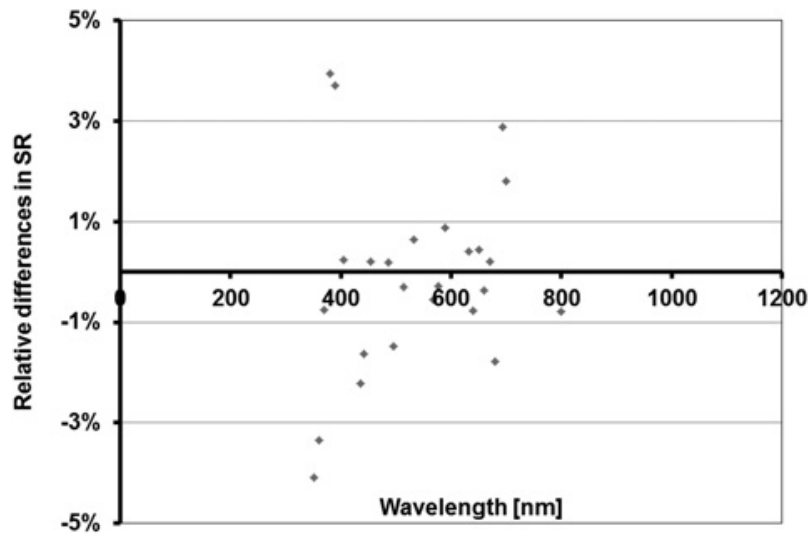

Fig. 12 Relative difference in spectral response of an a-Si device using the polychromatic filter method, compared to the monochromatic measurement 


\section{www.ietdl.org}
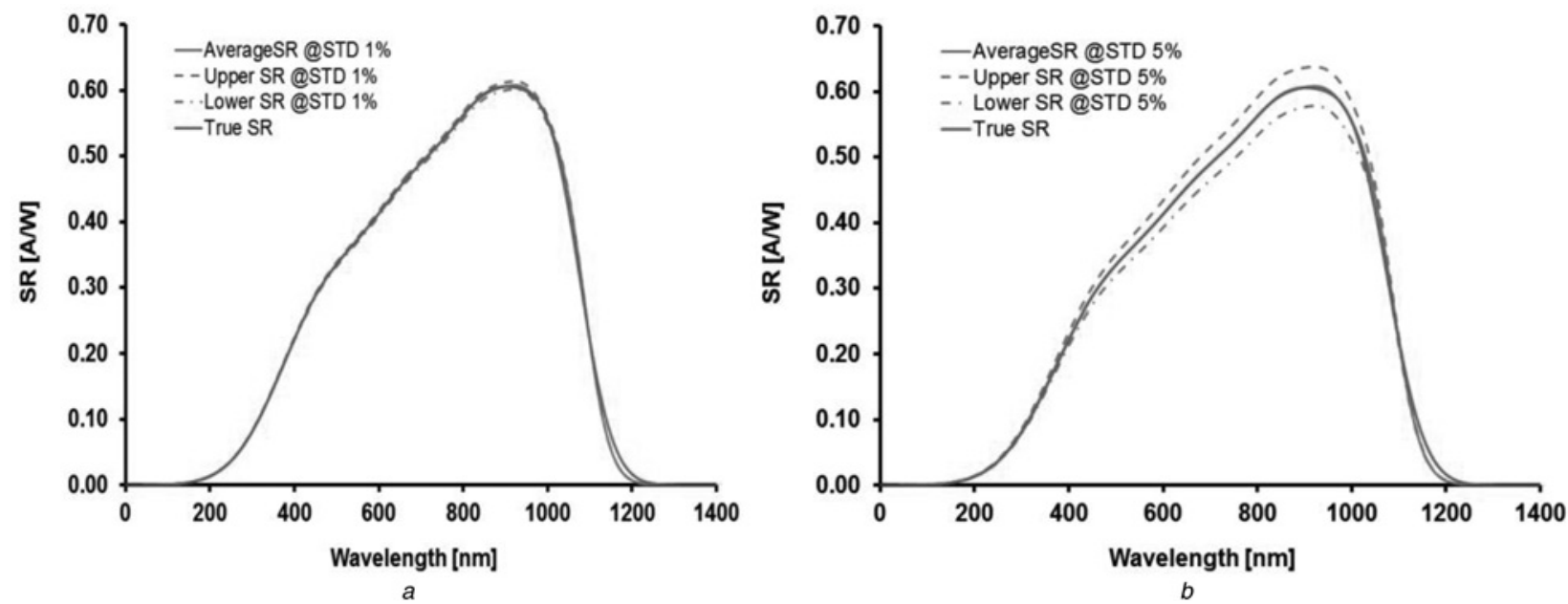

Fig. 13 Effect of uncertainty in the measured currents on the spectral response determination using the polychromatic filter method $a$ Uncertainty of $1 \%$ in the current measurement $b$ Uncertainty of $5 \%$ in the current measurement

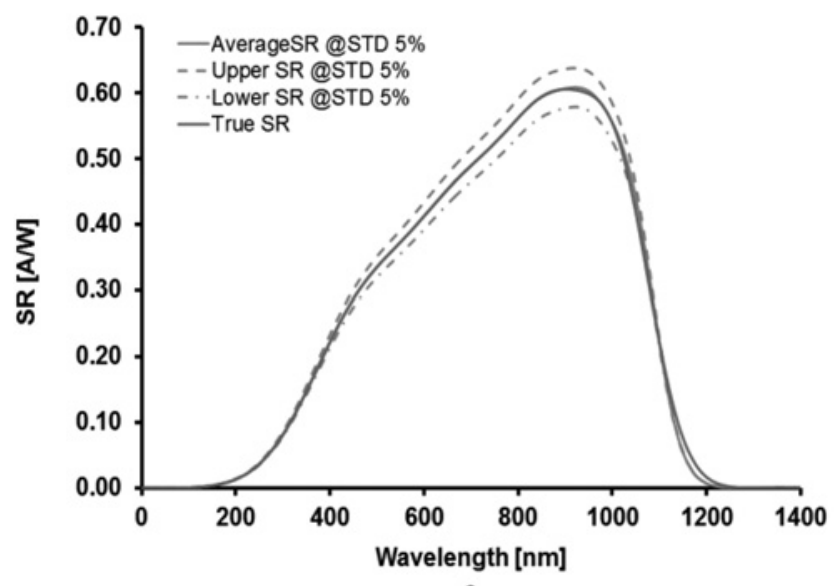

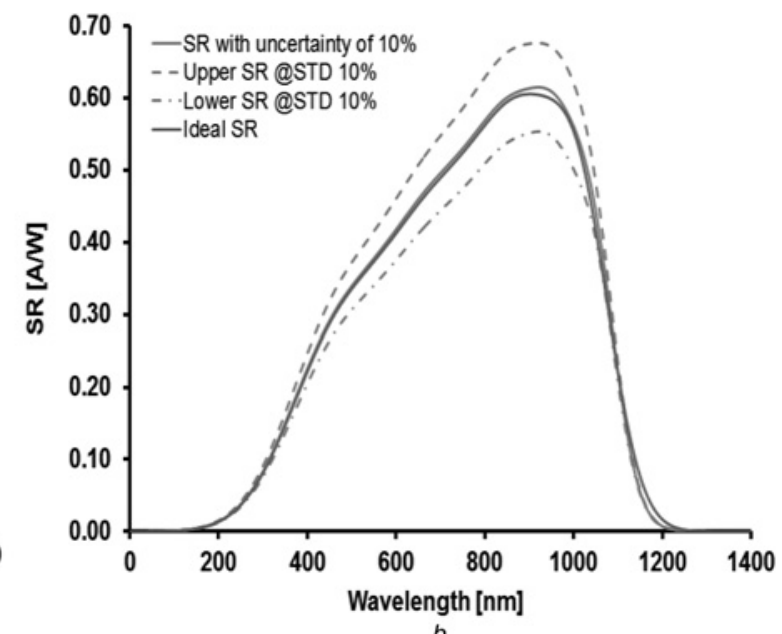

Fig. 14 Effect of uncertainty in the measured spectra on the spectral response determination using the polychromatic filter method $a$ Uncertainty of 5\% in the spectral measurement $b$ Uncertainty of $10 \%$ in the spectral measurement
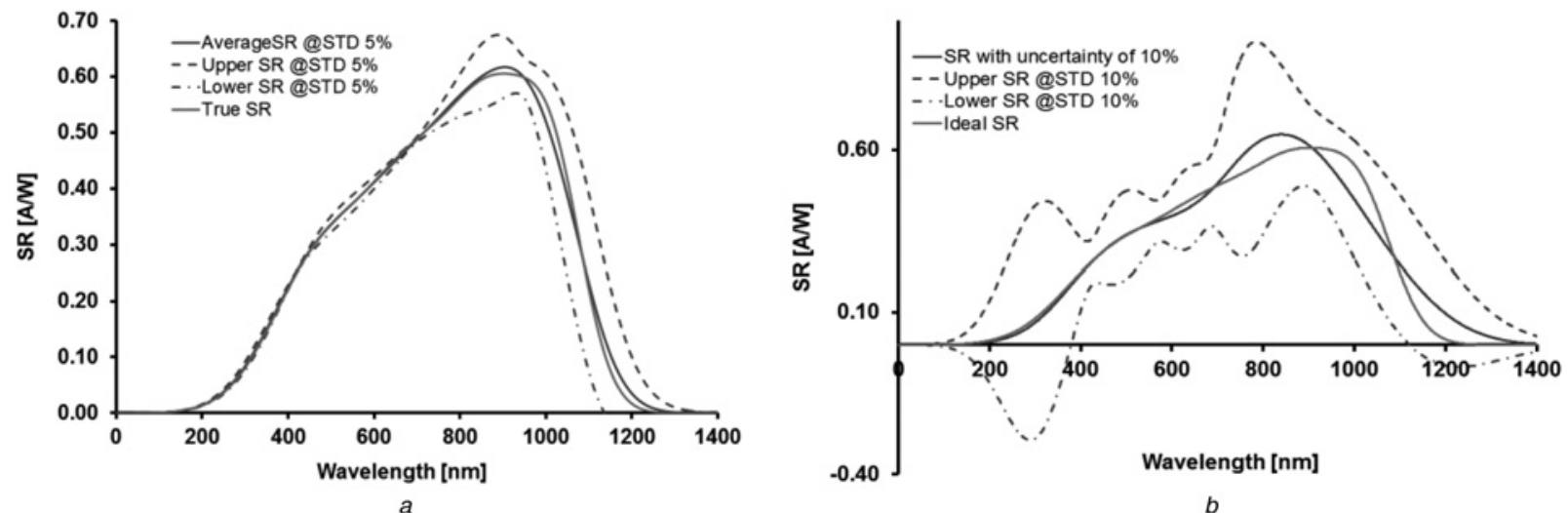

Fig. 15 Effect of uncertainty in the initial model coefficient values on the spectral response determination using the polychromatic filter method

$a$ Uncertainty of $5 \%$ in the chosen initial values

$b$ Uncertainty of $10 \%$ in the chosen initial values 
dependence of the accuracy on those input parameters can be determined.

The results of sensitivity analyses of the spectral response determination to individual input parameters are shown in the following figures.

As shown in Figs. 13-15, the accuracy of the results may be influenced by bias error in either current or spectral irradiance measurements and by choosing inappropriate initial values for the coefficients of the spectral response model. There is little influence of noise or random error in the measurement components. Measurement bias is relatively straightforward to minimise through careful calibration and use of instrumentation and most of the risk to the method arises from the model coefficient initial values. Although not trivial, this is a generic problem in all fitting/optimisation tasks and there is a large body of literature of solutions for both linear and non-linear models.

\section{Conclusions}

The proposed polychromatic filter method to determined PV device spectral response has been demonstrated as a feasible alternative to monochromatic methods. An empirical spectral response model based on a fifth-order Gaussian function has been used in the proposed method to obtain the spectral response of different PV devices. Although offering no insight into the device physics, it has been shown able to accommodate different shaped features of some diverse spectral response curves and presents an end result no less valid than any existing method.

Provided that instrumentation is carefully calibrated to eliminate bias, the final output of the method is robust against measurement noise or random uncertainties and there are no new challenges in the selection of appropriate starting values for the fitting process, nor shortage of methods to verify the fitting results.

The key advantages the proposed method offers over those existing are reduction of the complexity and cost of spectral response measurement, potentially reduction in the final uncertainty of the characteristic (still to be fully explored), and scalability to full-sized PV modules because of the removal of the lock-in requirement and overall higher photocurrent generation (currently the focus of on-going work in this area at CREST).

\section{Acknowledgment}

This work has been supported by a joint UK-India initiative in solar energy through a joint project 'Stability and Performance of Photovoltaics (STAPP)' funded by the Research Councils UK (RCUK) Energy Programme in the UK (contract no: EP/H040331/1) and by the Department of Science and Technology (DST) in India.

\section{References}

1 Hegedus, S.S., Shafarman, W.N.: 'Thin-film solar cells: device measurement and analysis', Prog. Phovolt., Res. Appl., 2004, 12, pp. $155-176$
2 Hishikawa, Y., Tsuno, Y., Kurokawa, K.: 'Spectral response measurements of PV modules and multi-junction devices' Proc. of the 22nd EUPVSEC, Milano, Italy, September 2007, pp. $2765-2769$

3 IEC60904-7: 'Computation of the spectral mismatch correction for measurements of photovoltaic devices', 2009

4 Mullejans, H., Ioannides, A., Kenny, R., Zaaiman, W., Ossenbrink, H., Dunlop, E.D.: 'Spectral mismatch in calibration of photovoltaic reference devices by global sunlight method', Meas. Sci. Technol., 2005, 16, pp. 1250-1254

5 Emery, K.: 'Measurement and characterization of solar cells and modules', in Luque, A., Hegedus, S. (Eds.): 'Handbook of photovoltaic Science and Engineering' (John Wiley \& Sons, Chichester, 2003), pp. 738-745

6 IEC60904-8: 'Measurement of Spectral Response of a Photovoltaic (PV) Device', 2007

7 Pravettoni, M., Komlan, A., Galleano, R., Mullejans, H., Dunlop, E.D.: 'An alternative method for spectral response measurements of large area thin-film photovoltaic modules', Prog. Photovolt: Res. Appl., 2012, 20, pp. 416-422

8 Mullejans, H., Bossong, H., Dunlop, E.D.: 'Temperature and bias light dependence of spectral response measurements'. Proc. of the PV Technology to Energy Solutions, Rome, Italy, October 2002, pp. 891-894

9 Mullejans, H., Wagner, T., Merli, F., Jager-Waldau, A., Dunlop, E.D.: 'Changes in spectral response with temperature and irradiance intensity', Thin Solid Films, 2004, 451-452, pp. 145-151

10 Holh-Ebinger, J., Warta, W.: 'Uncertainty of the spectral mismatch correction factor in STC measurements in photovoltaic devices', Prog. Photovolt., Res. Appl., 2011, 19, pp. 573-579

11 Emery, K., Dunlavy, D., Moriarty, T.: 'Photovoltaic spectral responsivity measurements'. Proc. of the Second World Conf. and Exhibition on Photovoltaic Solar Energy Conversion, Vienna, Austria, July 1998 , pp. 2298-2301

12 Kreinin, L., Bordin, N., Eisenberg, Y., Eisenberg, N.: 'Significance of bias light spectral composition for accurate measurement of silicon solar cell spectral response'. Proc. of the 33rd IEEE PVSC, San Diego, CA, USA, 11-16 May 2008, pp. 1329-1332

13 Field, H.: 'Solar cell spectral response measurement errors related to spectral band width and chopped light waveform'. Proc. of the 26th IEEE PVSC, Anaheim, CA, 30 September-3 October 1997, pp. $471-474$

14 Hartman, J.S., Lind, M.A.: 'Spectral response measurements for solar cells', Solar Cells, 1982, 7, pp. 147-157

15 Pravettoni, M., Nicola, M., Friesen, G.: 'A filtered pulsed solar simulator for spectral response measurements of multi-junction modules of commercial size'. Proc. of the 27nd EUPVSEC, Frankfurt, Germany, September 2012, pp. 3209-3213

16 Hohl-Ebinger, J., Warta, W.: 'Investigation of large area cell and module spectral response measurement'. Proc. of the 19th EUPVSEC, Paris, France, 7-11 June 2004, pp. 2611-2614

17 Pravettoni, M., Virtuani, A., Mullejans, H., Kenny, R.P., Dunlop, E.D., Barnham, K.W.J.: 'Spectral response measurements of multi-junction PV modules at the European solar test installation laboratories and their usage in clear day outdoor characterisation' Proc. of the 23rd EUPVSEC, Valencia, Spain, September 2008, pp. 2908-2912

18 Sara, I.D., Betts, T.R., Gottschalg, R.: 'Analysis of a suitable model for spectral response of a photovoltaic device'. Proc. of the 27nd EUPVSEC, Frankfurt, Germany, September 2012, pp. 471-475

19 Hibberd, C.J., Bliss, M., Upadhyaya, H.M., Gottschalg, R.: 'Characterisation of a filter-based external quantum efficiency measurement system'. Proc. of the Fifth PVSAT, Wrexham, UK, 2009, pp. 239-242

20 Hibberd, C.J., Plyta, F., Monokroussos, C., Bliss, M., Betts, T.R., Gottschalg, R.: 'Voltage-dependent quantum efficiency measurements of amorphous silicon multi-junction mini-modules', Sol. Energy Mater. Sol. Cells, 2011, 95, pp. 123-126 